\author{
V.I. SHALACK
}

\title{
On the Definitional Embeddability of Some Elementary Algebraic Theories into the First-Order Predicate Calculus
}

\author{
Shalack Vladimir Ivanovich \\ Department of Logic, Institute of Philosophy, Russian Academy of Sciences. \\ 14/5 Volkhonka St., Moscow, 119991, Russian Federation. \\ E-mail: shalack@gmail.com
}

In this article we prove a theorem on the definitional embeddability into first-order predicate logic without equality of such well-known mathematical theories as group theory and the theory of Abelian groups. This result may seem surprising, since it is generally believed that these theories have a non-logical content. It turns out that the central theory of general algebra are purely logical. Could this be the reason that we find them in many branches of mathematics? This result will be of interest not only for logicians and mathematicians but also for philosophers who study foundations of logic and its relation to mathematics.

Keywords: elementary theory, algebraic theory, groups, Abelian groups, definitions, definitional embedding, predicate logic

We assume that the language of the first-order predicate calculus is defined in the standard way as the set of terms and formulas over signature $\Sigma$, which consists of nonlogical relational and functional symbols. We write $L(\Sigma)$ for the first-order language with signature $\Sigma$. Models are pairs $M=\langle D, I\rangle$, where $D$ is a non-empty set of individuals, and $I$ is an interpretation of function and predicate symbols in the domain $D$. The relations $M, g \models A-$ "formula $A$ is true in the model $M$ with assignment of values to individual variables $g$ " and $M=A$ - "formula $A$ is true in the model $M$ " are defined in standard way.

\section{Defining new predicate symbols}

We can use definitions in order to extend the language $L(\Sigma)$ of theories with new predicate symbols. The definitions have the form of equivalences [1, p. 15]:

$$
\forall x_{1} \ldots x_{n}\left(P\left(x_{1}, \ldots, x_{n}\right) \equiv A\left(x_{1}, \ldots, x_{n}\right)\right)
$$

The definition must satisfy the conditions: 
1. $P \notin \Sigma$.

2. $A\left(x_{1}, \ldots, x_{n}\right) \in L(\Sigma)$.

3. The variables $x_{1}, \ldots, x_{n}$ are pairwise distinct.

4. The set of free variables of $A\left(x_{1}, \ldots, x_{n}\right)$ is included into $\left\{x_{1}, \ldots, x_{n}\right\}$.

After defining of the new predicate symbol $P$ it must be added to the signature $\Sigma$. As a result, there is a transition from the language $L(\Sigma)$ to the language $L(\Sigma \cup\{P\})$.

\section{The notion of definitional embedding}

Definition 1. The first-order theory $T$ in a language $L(\Sigma)$ with finite set of non-logical axioms $A x$ is definitionally embeddable into predicate calculus iff there are a signature $\Sigma^{\prime}$ and a set of definitions $D T$ of symbols $\Sigma \backslash \Sigma^{\prime}$ by formulas of $L\left(\Sigma^{\prime}\right)$ which met the following condition:

$$
\text { if } A \in L(\Sigma) \text { then } A x \vdash A \Leftrightarrow D T \vdash A \text {. }
$$

This definition is some variant of the notion of definitional embeddability of theories which was proposed by V.A. Smirnov [3].

\section{The elementary theories of groups, Abelian groups, and fields}

The theory of groups is formulated in the language over signature $\Sigma=$ $\{0,+,=\}$ where " 0 " is individual constant, " + " is 2 -ary functional symbol and " $="$ is 2 -place predicate.

The elementary theory of groups has the next non-logical axioms [2, p. 71].
A. $\forall x y z(x+(y+z)=(x+y)+z)$
B. $\forall x(0+x=x)$
C. $\forall x \exists y(y+x=0)$
D. $\forall x(x=x)$
E. $\forall x y(x=y \supset y=x)$
F. $\forall x y z(x=y \supset(y=z \supset x=z))$ 
G. $\forall x y z(y=z \supset(x+y=x+z \& y+x=z+x))$.

The elementary theory of Abelian groups needs additional axiom of commutativity [2, p. 71]:

H. $\forall x y(x+y=y+x)$.

THEOREM 1. The elementary theories of groups and Abelian groups are definitionally embeddable in the first order predicate calculus without equality.

Proof. We assume that the signature of the first-order predicate calculus contains the individual constant " 0 ", the 2-ary functional symbol " + ", and the binary predicate symbol " $\approx$ " but doesn't contain " =".

We accept the following definition

$(D G) \quad \forall x y(x=y \equiv(A \& \ldots \& G)[\approx /=] \supset x \approx y)$.

The expression $(A \& \ldots \& G)[\approx /=]$ denotes conjunction of the formulas $A-G$ in which the symbol " $=$ " is renamed into " $\approx$ ". And when it is necessary instead of $(A \& \ldots \& G)[\approx /=]$ we will use the shorthand $A G \approx$.

Let's check $D G \vdash A \& \ldots \& G$.

A. $\forall x y z(x+(y+z)=(x+y)+z)$

1. $\vdash A G \approx \supset A[\approx /=]$

2. $\vdash A G \approx \supset \forall x y z(x+(y+z) \approx(x+y)+z)-$ from 1

3. $\vdash \forall x y z(A G \approx \supset x+(y+z) \approx(x+y)+z)-$ from 2 , because $A G^{\approx}$ is closed

4. $D G \vdash \forall x y z(x+(y+z)=(x+y)+z) \quad-$ from 3 by $D G$

B. $\forall x(0+x=x)$

1. $\vdash A G \approx \supset B[\approx /=]$

2. $\vdash A G \approx \supset \forall x(0+x \approx x) \quad-$ from 1

3. $\vdash \forall x(A G \approx \supset 0+x \approx x) \quad-$ from 2

4. $D G \vdash \forall x(0+x=x) \quad-$ from 3 by $D G$

C. $\forall x \exists y(y+x=0)$ 
1. $\vdash A G \approx \supset C[\approx /=]$

2. $\vdash A G \approx \supset \forall x \exists y(y+x \approx 0) \quad$ - from 1

3. $\vdash \forall x \exists y(A G \approx \supset y+x \approx 0) \quad$ - from 2

4. $D G \vdash \forall x \exists y(y+x=0) \quad$ - from 3 by $D G$

D. $\forall x(x=x)$

1. $\vdash A G \approx \supset D[\approx /=]$

2. $\vdash A G \approx \supset \forall x(x \approx x) \quad$-from 1

3. $\vdash \forall x(A G \approx \supset x \approx x) \quad-$ from 2

4. $D G \vdash \forall x(x=x) \quad-$ from 3 by $D G$

E. $\forall x y(x=y \supset y=x)$

1. $\vdash A G \approx \supset E[\approx /=]$

2. $\vdash A G \approx \supset \forall x y(x \approx y \supset y \approx x) \quad$ - from 1

3. $\vdash \forall x y(A G \approx \supset(x \approx y \supset y \approx x)) \quad-$ from 2

4. $\vdash \forall x y\left((A G \approx \supset x \approx y) \supset\left(A G^{\approx} \supset y \approx x\right)\right)-$ from 3 by self-distr. $\supset$

5. $D G \vdash \forall x y(x=y \supset y=x) \quad-$ from 4 by $D G$

F. $\forall x y z(x=y \supset(y=z \supset x=z))$

1. $\vdash A G \approx \supset F[\approx /=]$

2. $\vdash A G \approx \supset \forall x y z(x \approx y \supset(y \approx z \supset x \approx z)) \quad$ - from 1

3. $\vdash \forall x y z(A G \approx \supset(x \approx y \supset(y \approx z \supset x \approx z))) \quad$ - from 2

4. $\vdash \forall x y z((A G \approx \supset x \approx y) \supset(A G \approx \supset$

$(y \approx z \supset x \approx z)))-$ from 3 by self-distr. $\supset$

5. $\vdash \forall x y z((A G \approx \supset x \approx y) \supset((A G \approx \supset y \approx z) \supset$

$(A G \approx \supset x \approx z)))-$ from 4

6. $D G \vdash \forall x y z(x=y \supset(y=z \supset x=z)) \quad-$ from 5 by $D G$

G. $\forall x y z(y=z \supset(x+y=x+z \& y+x=z+x))$

1. $\vdash A G \approx \supset G[\approx /=]$

2. $\vdash A G \approx \supset \forall x y z(y \approx z \supset(x+y \approx x+z \& y+x \approx z+x)) \quad$ - from 1 


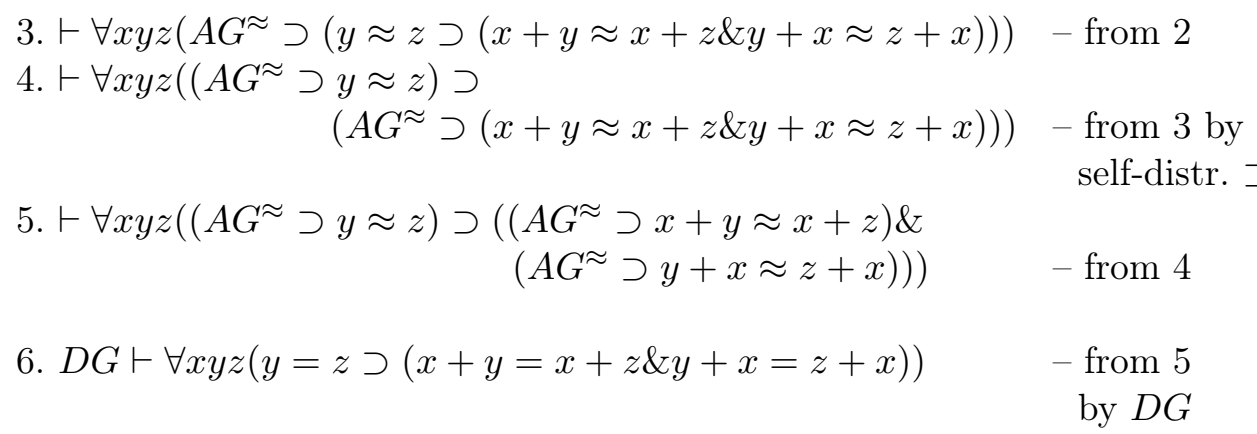

We have shown that for every formula $U \in L_{T G}$ if $A \& \ldots \& G \vdash_{T G} U$ then $D G \vdash U$.

For the Abelian groups we introduce the following definition:

$(D A) \quad \forall x y(x=y \equiv(A \& \ldots \& H)[\approx /=] \supset x \approx y)$.

The expression $(A \& \ldots \& H)[\approx /=]$ denotes conjunction of the formulas $A-H$, in which the symbol " $=$ " is renamed into " $\approx$ ". And when it is necessary instead of $(A \& \ldots \& H)[\approx /=]$ we will use the shorthand $A H^{\approx}$.

Let's check $D A \vdash A \& \ldots \& H$.

H. $\forall x y(x+y=y+x)$

1. $\vdash A H \approx \supset H[\approx /=]$

2. $\vdash A H \approx \supset \forall x y(x+y \approx y+x)$

- from 1

3. $\vdash \forall x y(A H \approx \supset x+y \approx y+x)$

- from 2

4. $D A \vdash \forall x y(x+y=y+x)$

- from 3 by $D A$

Thus, we have shown that for every formula $U \in L_{T G A}$ if $A \& \ldots \& H \vdash_{T G A} U$ then $D A \vdash U$.

It remains to show that for every formula $U \in L_{T G / T G A / T F}$ if $D G / D A \vdash U$ then $\vdash_{T G / T G A} U$. The proof is the same for all theories. We present the proof of $T G$.

Let us assume, that $D G \vdash U$ but not $A \& \ldots \& G \vdash U$. By the completeness theorem of the first-order predicate logic it follows, that $D G \models U$, and there exists such a model $M=\langle D, I\rangle$ of theory $T_{G}$ and an assignment of values to individual variables $g$ that $M \models A \& \ldots \& G$ and $M, g \mid \neg U$. 
We can extend the model $M=\langle D, I\rangle$ to the model $M^{\prime}=\left\langle D, I^{\prime}\right\rangle$ in which the formula $D G$ is true. It is sufficient to expand the domain of the function $I$ so that the new function of interpretation $I$ ' ascribes value $I^{\prime}(\approx)=I(=)$ to predicate symbol " $\approx$ ", and for all other functional and predicate symbols it retains the same values as $I$.

Since $M \models A \& \ldots \& G$, then in the model $M^{\prime}=\left\langle D, I^{\prime}\right\rangle$ by the definition of $I^{\prime}$ we will have $M^{\prime} \mid=(A \& \ldots \& G)[\approx /=]$ and hence $M^{\prime}=$ $x_{1}=x_{2} \equiv\left((A \& \ldots \& G)[\approx /=] \supset x_{1} \approx x_{2}\right)$. It follows that $D G$ is true in the model $M^{\prime}$. Therefore by our assumption $D G \models U$ it must be $M^{\prime}, g \models U$. However, the formula $U$ does not contain the symbol " $\approx$ ", while all other descriptive symbols are interpreted in the same way as in the model $M$, and by our assumption $M, g \models \neg U$ it must be $M^{\prime}, g \models \neg U$. We have obtained the contradiction. Therefore the assumption that $A \& \ldots \& G \vdash U$ does not hold, is false.

\section{Comments}

The theorem contradicts to our usual conviction that listed therein elementary algebraic theories carry some non-logical information about their models. That's not so. It turns out that we can use the language of the first-order predicate calculus, and without resorting to the postulation of non-logical axioms, to build these theories with help of usual definitions.

This result is the consequence of a more general theorem on sufficient conditions for theories be definitionally embeddable into the first-order predicate calculus. The general theorem will be published in the near future, but we can already say that it has a lot of effects that force for a fresh look at the many well-known theories.

\section{References}

[1] Karpovich, V.N. Terminy v strukture teorii. Logicheskij analiz [ Terms in the structure of the theory. Logical analysis]. Novosibirsk: Nauka, 1978. 128 pp. (In Russian)

[2] Mendelson, E. Vvedenie v matematicheskuyu logiku [Introduction to Mathematical Logic], 4-th ed., M.: Nauka, 1997. 440 pp. (In Russian)

[3] Smirnov, V.A. "Logical Relations between Theories", Synthese, 1986, 66(1), pp. $71-87$.

[4] Shalack, V. "On Some Applied First-Order Theories which Can be Represented by Definitions", Bulletin of the Section of Logic, 2015, 44/1-2, pp. 19-24. 